\title{
Clinical Evidence of Effective Therapy for Prostate Inflammation Based on a Combination of Macrolides and Men's Pads
}

\author{
Edvard V. Kryzhanovskii ${ }^{*}$, Alexander B. Yavorsky², Lim Kwong Choong3 \\ 1"Telebiomet” SIE of Moscow Technical University of Communications and Informatics, Saint-Petersburg, Russia \\ ${ }^{2}$ Sports Medicine Clinics of Russian State University of Physical Education, Sport, Youth and Tourism, Moscow, Russia \\ ${ }^{3}$ BAE International Inc. SDN BHD, Kuala-Lumpur, Malaysia \\ Email: ^edward@telebiomet.ru
}

How to cite this paper: Kryzhanovskii, E.V., Yavorsky, A.B. and Choong, L.K. (2021) Clinical Evidence of Effective Therapy for Prostate Inflammation Based on a Combination of Macrolides and Men's Pads. Modern Research in Inflammation, 10, 1-9. https://doi.org/ 10.4236/mri.2021.101001

Received: January 26, 2021

Accepted: February 23, 2021

Published: February 26, 2021

Copyright $\odot 2021$ by author(s) and Scientific Research Publishing Inc. This work is licensed under the Creative Commons Attribution International License (CC BY 4.0).

http://creativecommons.org/licenses/by/4.0/

(c) (i) Open Access

\begin{abstract}
Prostatitis is a widespread disease associated with pelvic pain syndrome, which is characterized by pelvic pain, various urinary symptoms, and possible sexual dysfunction. An adequate therapy requires schemes for chronic prostatitis that affects, among other things, the atypical causative agents. We investigate a therapy for prostate inflammation consisting in a combination of macrolides with the usage of men's pads for underwear during the treatment. We demonstrate the efficacy of this therapy on a group of patients with chronic prostatitis. The group of patients under this combination therapy exhibits better recovery dynamics compared to the group of patients who were treated only by macrolides. We observe a statistically significant (p-value $<$ 0.01 ) difference between the two groups in the data from three sections of the NIH-CPSI questionnaire, which concerned pain, symptoms of impaired urination, and quality of life. The number of patients with soreness of the prostate, with the presence of bacteria and leukocytes in the prostate gland secretion, and with the presence of microorganisms in the PCR tests after treatment was lower in the combination therapy group compared to the macrolide group. The obtained results demonstrate that the use of macrolides in combination with the use of men's pads in the treatment of chronic prostate inflammation, including "abacterial" prostatitis, is promising. More generally, our results highlight the importance of flexible and individual approaches to the treatment of chronic prostatitis.
\end{abstract}

\section{Keywords}

Prostatitis, Men's Pad, Combination Therapy 


\section{Introduction}

Chronic and acute inflammation of the prostate is the most common urological disease in men under the age of 50, while in older patients it ranks third in prevalence after benign prostatic hyperplasia and prostate cancer [1]. According to the recommendations of the European Association of Urology and the Russian Society of Urology, for the treatment of patients with chronic prostatitis and/or chronic pelvic pain syndrome, most urologists prescribe antimicrobial therapy with first-line drugs (fluoroquinolones) for 4 weeks [2]. This is often done empirically, without verifying the primary bacterial agent and determining its sensitivity.

Assessing the possibilities of using traditional bacteriological research and PCR diagnostics in the protocol for examining patients with chronic prostatitis, Y. S. Choi et al. showed that the atypical microorganisms (Chlamydia, Mycoplasma, and Ureaplasma) in combination with E. Coli, Ps. aeruginosa, and coccal flora were detected in $40 \%$ of cases [3]. Such a comprehensive study of the biomaterial for patients with chronic prostatitis is very rare in outpatient practice, and, therefore, an adequate therapy, as a rule, is not carried out. This is considered to be the reason for the inversion of bacterial prostatitis into "abacterial", and, as a result, the patient is becoming incurable. Dissatisfaction with treatment and frequent relapses of the disease often force patients to go out of control or change doctors, which leads to further chronic inflammation with worse prognosis and appearance of complications.

The above points prompted the need to investigate the effectiveness of the use of alternative therapy regimens for chronic prostatitis, affecting, among other things, the atypical causative agents of prostatitis. It was shown that the usage of specific facilities made of special materials, such as special bra pads for women, in the everyday life of a patient may promote accelerated normalization in the urogenital and endocrine systems [4]. In a series of papers, the authors of this study reported clinical data demonstrating how the usage of various biomedical products based on a similar technology as the mentioned bra pads improves the patient recovery in various diseases [5]-[10]. Here, we investigate the efficacy of a therapy for the treatment of men with chronic prostatitis based on a combination of macrolides with men's pads for underwear (hereinafter referred to as MPAD), which have the same production and technology as the bra pads from [4]. We show that the usage of such MPAD during the treatment improves the disease dynamics.

\section{Study Design}

The study was carried out in the Sports Medicine Clinics of the Russian State University of Physical Education, Sport, Youth, and Tourism (Moscow, Russia), in collaboration with the small innovative company "Telebiomet" from the Moscow Technical University of Communications and Informatics. It was officially approved by the Hospital Ethical Commission. 
The study group included 20 men with chronic prostatitis, whose demographic, clinical, and laboratory data were assessed. The average age of the patients was $35 \pm 18$ years. Patients with comorbidities were not included in the study group, and that was the only constraint on participation in the study. All participants were Russians and citizens of Moscow and belonged to various social groups in terms of income. According to the statistical analysis plan, the patients were divided into two groups of therapy: patients from the first group were treated only by a macrolide (10 patients), and patients from the second group underwent a combination therapy (10 patients). According to the combination therapy, patients from the second group both received a macrolide and, in parallel, wore MPAD in an underwear pocket from 2 to 5 weeks during the therapy (according to the decision of the attending physician). The MPAD appearance is shown in Figure 1, and Figure 2 demonstrates the scheme of its disposition. The composition of MPAD is identical to the bra pads from [4]. Josamycin was used as a macrolide. The treatment data for the patients were collected at the beginning (visit 1 to the doctor) and after treatment (visit 2).

The patients were included in the study after the diagnosis of the prostate inflammation and the urologist's decision to prescribe macrolide for the treatment of prostatitis, subject to the signing of an informed consent to participate in the program. The treatment regimens were determined by the doctor and corresponded to the recommended ones, according to the instructions for medical use (500 mg of josamycin, 3 times a day).

The scope of examination of patients corresponded to the usual examination in accordance with the Standard of Care for Patients with Prostatitis. During the first and repeated visits, the following were carried out.

- registration of complaints and anamnesis in accordance with the Chronic Prostatitis Symptom Scale Index (NIH-CPSI);

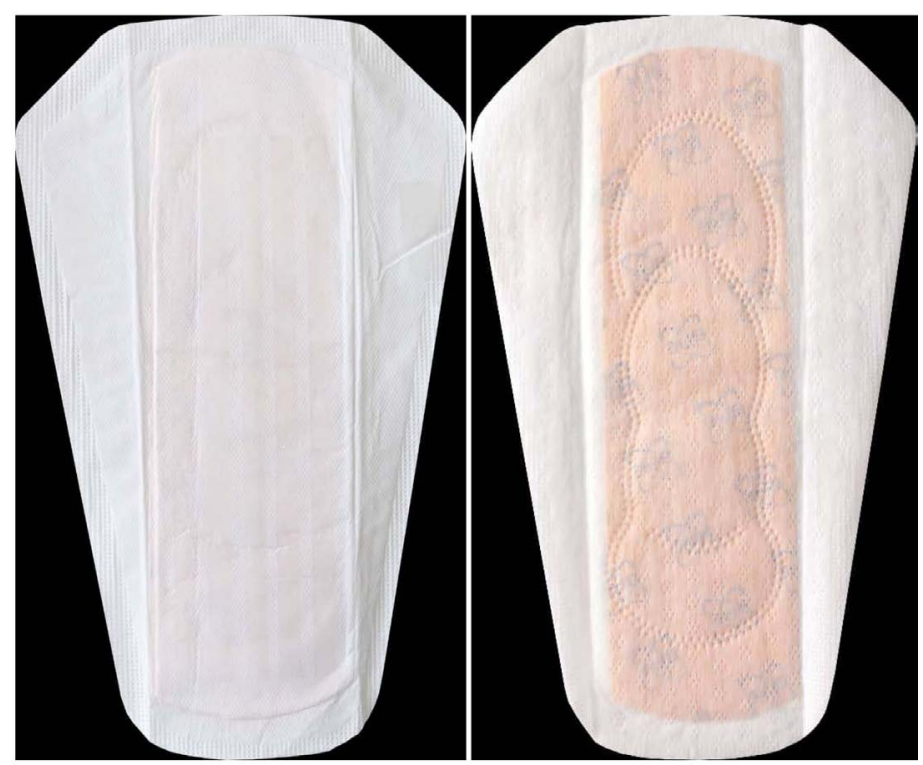

Figure 1. MPAD appearance (outside on the left, inside on the right). 

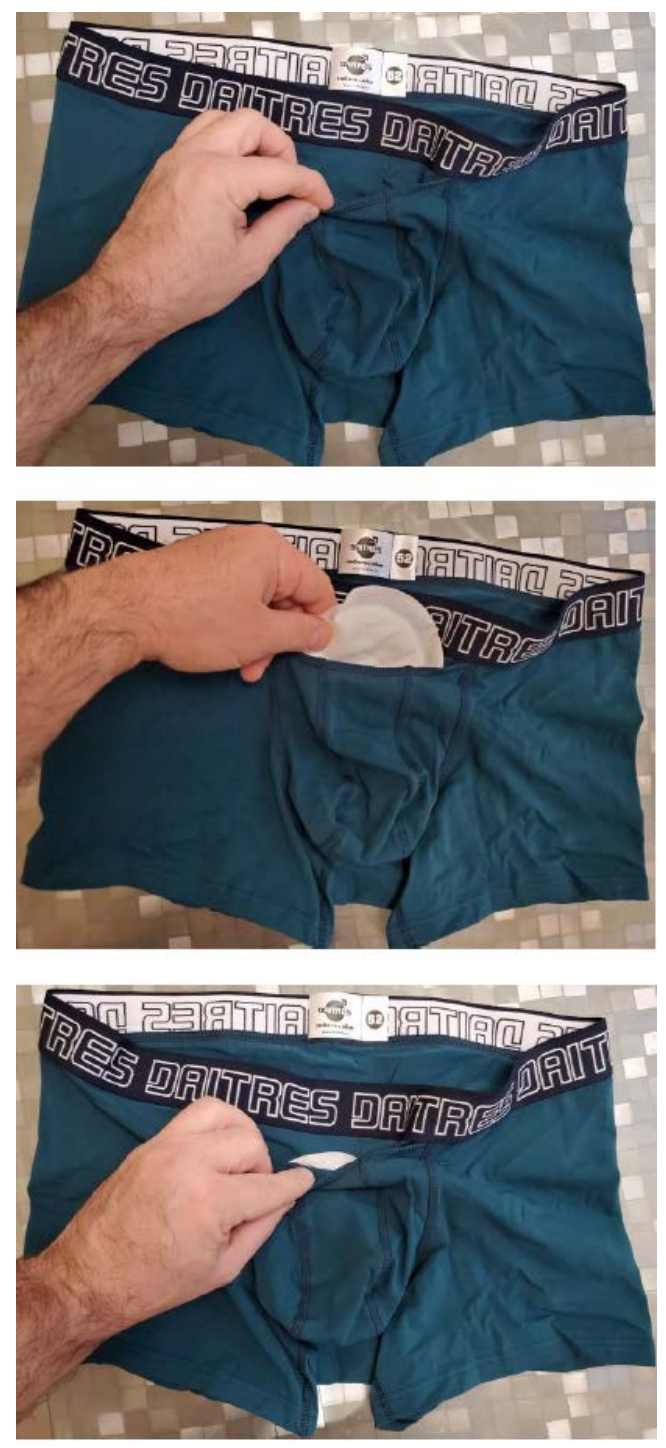

Figure 2. Disposition of MPAD in an underwear pocket.

- digital rectal examination (assessment of the volume, pain, and density of the prostate gland);

- ultrasound examination of the kidneys, bladder, and prostate gland (with registration of the volumes of residual urine and prostate gland);

- general analysis of the prostate gland secretion (content of bacteria and leukocytes).

Based on the dynamics of changes in the above parameters, the urologist assessed the effectiveness of the patient's treatment.

The physician assessed the efficacy of macrolides and tetracyclines at visit 2, indicating one of the following treatment outcomes.

- recovery (complete resolution of clinical signs and symptoms);

- improvement (partial resolution of signs and symptoms);

- lack of effect (inadequate response to therapy and the need for additional antibiotic treatment); 
- impossible to assess (patient is lost to follow-up).

In the course of the study, we collected and analyzed data from three sections of the NIH-CPSI questionnaire, which concerned pain, symptoms of impaired urination, and quality of life (Figure 3). The total score as a sum of all points in these three sections was recorded for each patient before and after the treatment (visits 1 and 2 to the doctor, respectively). Smaller values of the score correspond to better health conditions. Statistical analysis was carried using the Statistica software.

\section{Results}

We analyzed the dynamics of the total score from the NIH-CPSI questionnaire

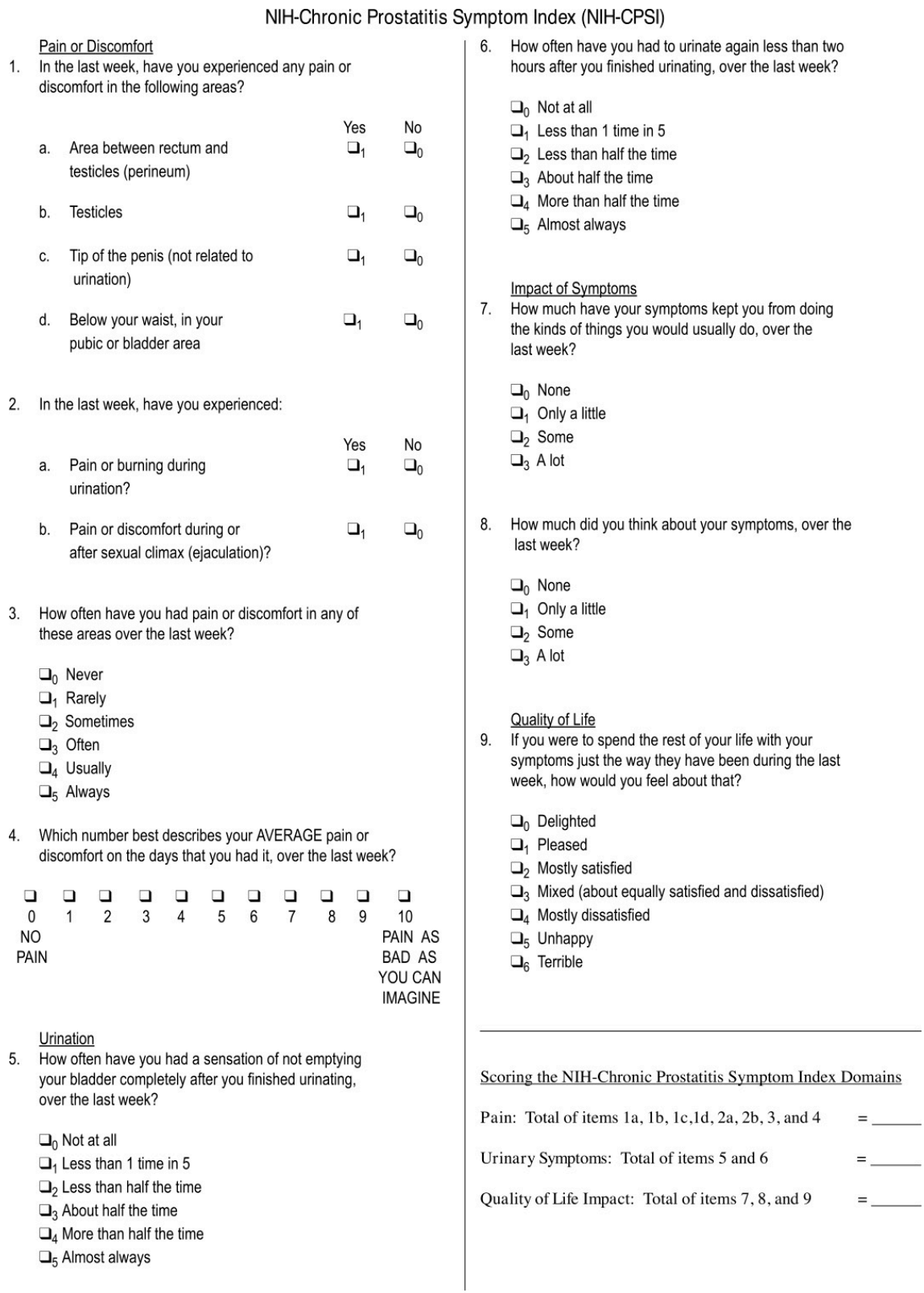

Figure 3. NIH-CPSI questionnaire. 
for each patient and compared these dynamics between the two groups of patients (Figure 4). In the macrolide therapy group ("no-MPAD" group), the baseline average score of $25 \pm 3$ decreased to $8 \pm 2$ after treatment. In the combination therapy group ("MPAD" group), the average score was $26 \pm 2$ before treatment and $5 \pm 1$ after treatment. The analysis showed a statistically significant difference between the scores in the two groups after the treatment ( $\mathrm{p}$-value $<$ 0.01) (Figure 4). Thus, based on the NIH-CPSI questionnaire, patients who used MPAD during treatment demonstrated better dynamics of recovery than patients who did not use MPAD.

Table 1 shows how other parameters associated with the disease varied during treatment in the two groups.

Soreness of the prostate during the study significantly decreased in all treatment groups: the number of patients with soreness decreased from $80 \%$ to $30 \%$

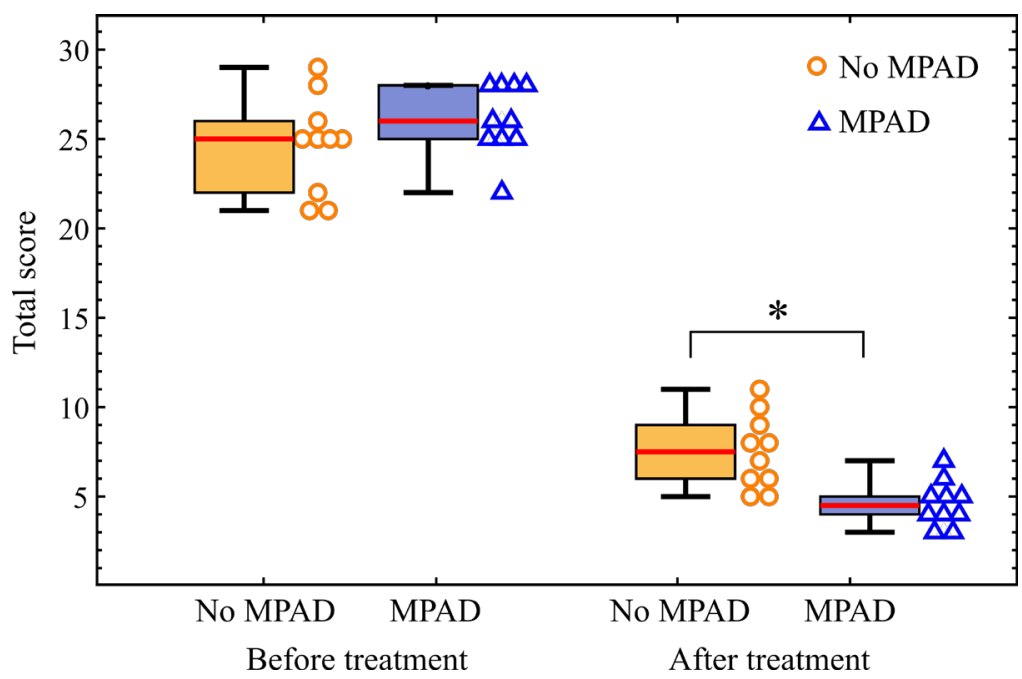

Figure 4. Boxplots and scatter diagrams of the total scores from the NIH-CPSI questionnaire in the MPAD and no-MPAD groups of patients at visits 1 (before treatment) and 2 (after treatment) to the doctor. The actual scores are shown for each group before and after treatment to the right of the boxplots. The asterisk marks a statistically significant difference between the average scores in the groups ( $\mathrm{p}$-value $<0.01$ according to the Mann-Whitney test).

Table 1. Dynamics of other parameters.

\begin{tabular}{ccccc}
\hline \multirow{2}{*}{$\begin{array}{c}\text { No. of patients with a } \\
\text { parameter }\end{array}$} & \multicolumn{2}{c}{ Before treatment } & \multicolumn{2}{c}{ After treatment } \\
\cline { 2 - 5 } & No-MPAD group & MPAD group & No-MPAD group & MPAD group \\
\hline Soreness & 8 & 9 & 3 & 1 \\
Bacteria & 7 & 7 & 2 & 0 \\
Leukocytes & 10 & 8 & 6 & 4 \\
Microorganisms (by PCR) & 5 & 7 & 2 & 0 \\
Recovered & - & - & 4 & 4 \\
Improved & - & - & 4 & 5 \\
\hline
\end{tabular}




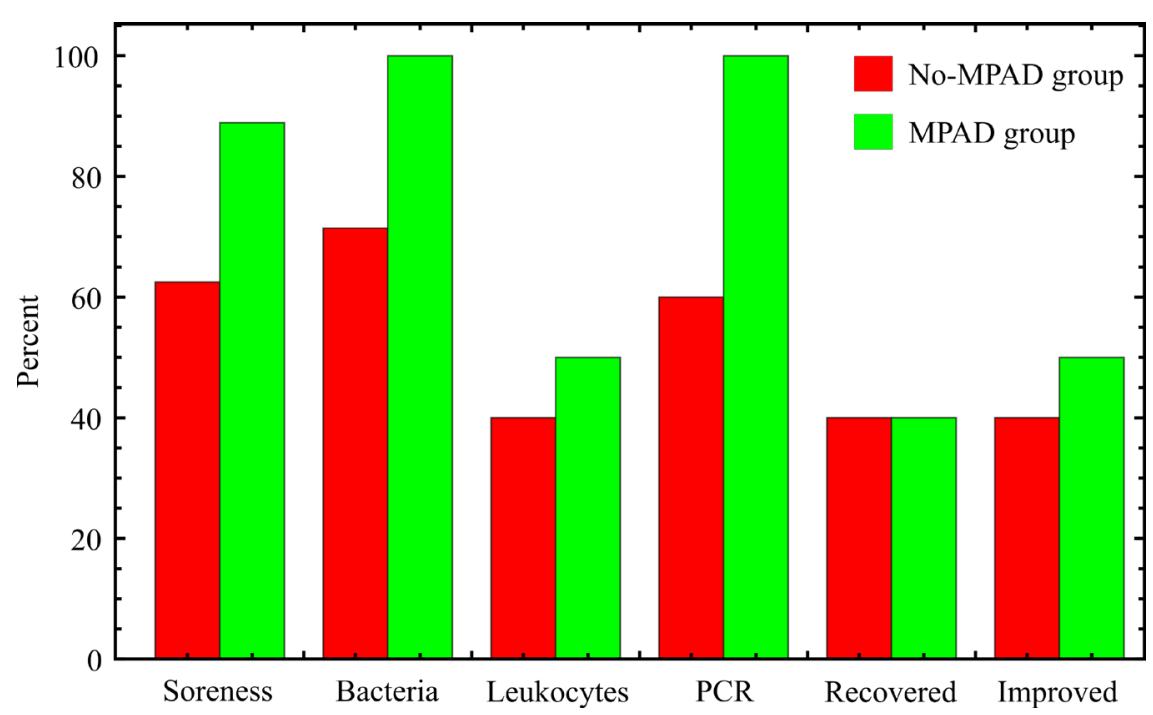

Figure 5. Difference between the numbers of patients with a given parameter before and after treatment in percent of the number of patients with this parameter before treatment. The parameters are from Table 1.

in the no-MPAD group and from $90 \%$ to $10 \%$ in the MPAD group. Laboratory examination of prostate secretion revealed the presence of bacteria in the secretion of the prostate gland from $70 \%$ patients within each group at visit 1 to the doctor. At visit 2, this number decreased to $20 \%$ in the no-MPAD group and no bacteria were detected in the MPAD group. Leukocytes in the secretion of the prostate gland were detected in $100 \%$ of patients in the no-MPAD group and $80 \%$ of patients in the MPAD group at visit 1 , and after treatment these numbers reduced to $60 \%$ and $40 \%$, respectively.

In studies using the PCR method, microorganisms were revealed from $50 \%$ of patients in the no-MPAD group and $70 \%$ of patients in the MPAD group at visit 1 , and these numbers reduced to $20 \%$ and $0 \%$, respectively, at visit 2 . Finally, the doctors estimated the outcome of the prescribed therapy as "recovery" in $40 \%$ of patients within each group. The "improvement" estimate was recorded for $40 \%$ of patients in the no-MPAD group and $50 \%$ of patients in the MPAD group.

Figure 5 shows the cumulative recovery rate for the two groups and for all parameters from Table 1, where by recovery rate we mean the difference between the numbers of patients with a given parameter before and after treatment as a percentage of the number before treatment. It can be seen that the group of patients who used MPAD demonstrates a faster recovery as compared with the no-MPAD group for all parameters except full recovery, for which the two groups are equivalent. This multi-parameter comparison confirms the difference between the groups in terms of the disease dynamics.

\section{Discussion and Conclusions}

The study demonstrated good evidence for the high efficacy of a combination of macrolides (Josamycin) and MPAD in men with chronic prostatitis in routine 
clinical practice. Overall, the therapy studied in this program, according to doctors, was effective in $90 \%$ of patients.

To estimate the effectiveness of therapy, modern and classical methods of assessing the condition of patients were used, as well as methods of statistical data analysis. Among the studied therapeutic groups, according to the data of the dynamic assessment of clinical laboratory and instrumental parameters, the most pronounced positive dynamics was observed in the combination therapy group. In this group, the quality of life significantly increased, the number of bacteria in the secretion of the prostate gland decreased, and the total recovery and improvement rates exceeded those in the macrolide monotherapy group.

Based on the obtained results, we may conclude that the use of macrolides in combination with the use of MPAD in the treatment of chronic prostate inflammation, including "abacterial" prostatitis, is promising. However, a rather small number of patients participated in our study is a disadvantage. For more definite conclusions, additional studies with larger samples are required.

In general terms, the study confirms the importance of a flexible and individual approach to the treatment of chronic prostatitis, the need for constant evaluation of the effectiveness of treatment, monitoring and a critical approach to existing therapy regimens, the importance of patient adherence to treatment and dynamic follow-up by a urologist in the framework of daily clinical practice.

\section{Acknowledgements}

Special thanks to Mr. Lim Fap Khoon of Kwong Hing Group for the continuous corporate support, to Professor Muslim Liew Bin Abdullah for the BAE research and development, and to Mr. Yuichiro Meitoma, Director of BAE Japan.

\section{Conflicts of Interest}

The authors declare no conflicts of interest regarding the publication of this paper.

\section{References}

[1] Nickel, J.C. (2011) Prostatitis. Canadian Urological Association Journal, 5, 306-315. https://doi.org/10.5489/cuaj.686

[2] Nickel, J.C., Downey, J., Hunter, D. and Clark, J. (2001) Prevalence of Prostatitis-like Symptoms in a Population Based Study Using the National Institutes of Health Chronic Prostatitis Symptom Index. Journal of Urology, 165, 842-845. https://doi.org/10.1016/S0022-5347(05)66541-X

[3] Choi, Y.S., Kim, K.S., Choi, S.W., Kim, S., Bae, W.J., Cho, H.J., Hong, S.H., Kim, S.W., Hwang, T.K. and Lee J.Y. (2013) Microbiological Etiology of Bacterial Prostatitis in General Hospital and Primary Care Clinic in Korea. Prostate International, 1, 133-138. https://doi.org/10.12954/PI.13023

[4] Kryzhanovskii, E.V., Yavorsky, A.B., Lim, K.C. and Liew Muslim, B.A. (2019) Breast State Improvement by Using the Lymphatic Drainage Disposable Bra Pads. International Journal of Research in Medical Sciences, 7, 1-5. https://doi.org/10.18203/2320-6012.ijrms20190925 
[5] Krizhanovsky, E.V., Tursunova, K., Lim, K.C. and Ng, C.P. (2009) The Hypoglycemic Properties of BAE Maca Max Preparation. Online Journal of Biological Sciences, 9, 17-20. https://doi.org/10.3844/ojbsci.2009.17.20

[6] Krizhanovsky, E.V. and Lim, K.C. (2014) Study of the Influence of Subtle Energetic Changes in Environment on the Productivity of the Process of Sleep. Open Journal of Ecology, 4, 693-702. https://doi.org/10.4236/oje.2014.411059

[7] Krizhanovsky, E.V., Lim, K.C. and Laskova, N. (2015) The Study of Natural Solution Protective Activity on H7N9 Virus. Journal of Biosciences and Medicines, 3, 1-6. https://doi.org/10.4236/jbm.2015.35001

[8] Krizhanovsky, E.V. and Lim, K.C. (2015) Simulazione Della Attività Di Protezione Naturale Preparazione on H7N9 Virus. Italian Science Review, 3, 1-5.

[9] Krizhanovsky, E.V., Yavorsky, A.B. and Lim, K.C. (2017) Influence of the Subtle Energetic Changes on the Treatment Response in Patients with Insomnia. Global Journal of Health Science, 9, 90-96. https://doi.org/10.5539/gihs.v9n9p90

[10] Krizhanovskii, E.V., Yavorsky, A.B. and Lim, K.C. (2018) The Sleep Quality of Patients with Insomnia under the Influence of Subtle Energy Fields. International Journal of Research in Medical Sciences, 6, 2193-2199.

https://doi.org/10.18203/2320-6012.ijrms20182802 